\title{
THE MINIMAL FAITHFUL PERMUTATION DEGREE FOR A DIRECT PRODUCT OBEYING AN INEQUALITY CONDITION
}

\author{
DAVID EASDOWN AND NEIL SAUNDERS
}

\begin{abstract}
The minimal faithful permutation degree $\mu(G)$ of a finite group $G$ is the least nonnegative integer $n$ such that $G$ embeds in the symmetric group $\operatorname{Sym}(n)$. Clearly $\mu(G \times H) \leq \mu(G)+\mu(H)$ for all finite groups $G$ and $H$. Wright (1975) proves that equality occurs when $G$ and $H$ are nilpotent and exhibits an example of strict inequality where $G \times H$ embeds in Sym(15). Saunders (2010) produces an infinite family of examples of permutation groups $G$ and $H$ where $\mu(G \times H)<\mu(G)+\mu(H)$, including the example of Wright's as a special case. The smallest groups in Saunders' class embed in Sym(10). In this paper we prove that 10 is minimal in the sense that $\mu(G \times H)=\mu(G)+\mu(H)$ for all groups $G$ and $H$ such that $\mu(G \times H) \leq 9$.
\end{abstract}

\section{INTRODUCTION}

Throughout this paper all groups are assumed to be finite. The minimal faithful permutation degree $\mu(G)$ of a group $G$ is the smallest nonnegative integer $n$ such that $G$ embeds in the symmetric group $\operatorname{Sym}(n)$. Recall that the core of a subgroup $H$ of $G$, denoted by $\operatorname{core}(H)$, is the largest normal subgroup of $G$ contained in $H$, and that $H$ is core-free if core $(H)$ is trivial. Thus $\mu(G)$ is the smallest sum of indexes for a collection of subgroups $G_{1}, \ldots, G_{\ell}$ of $G$ such $\cap_{i=1}^{\ell} G_{i}$ is core-free. The subgroups $G_{i}$ are the respective point-stabilisers for the action of $G$ on its orbits and letters in the $i$ th orbit may be identified with cosets of $G_{i}$. If $\ell=1$ then the representation is transitive and $G_{1}$ is a core-free subgroup.

For any groups $G$ and $H$, we always have the inequality

$$
\mu(G \times H) \leq \mu(G)+\mu(H) .
$$

Johnson and Wright (see $[6,10]$ ) developed a general theory of minimal degrees of groups and described conditions for when equality occurs in (1). They proved this to be the case when $G$ and $H$ have coprime orders and when $G$ and $H$ are nilpotent. Easdown and Praeger (see [5]) showed that equality holds when $G$ and $H$ are direct products of simple groups. Wright in [10] asked whether equality occurs in (1) always and an example exhibiting strict inequality was attached as an addendum, where $G$ and $H$ are given as subgroups of Sym(15). In that example, $G$ and $H$ generate a subgroup $G H$ of $\operatorname{Sym}(15)$ that is an internal direct product of $G$ and $H$.

Saunders showed in [7] that the example in [10] fits into a general family that provides infinitely many instances of strict inequality in (1). There $G$ could be taken to be the complex reflection group $G(p, p, q)$, where $p$ and $q$ are distinct odd primes satisfying certain

AMS subject classification (2010): 20B35.

Keywords: Faithful Permutation Representations. 
other conditions, and $H$ the centraliser of the minimally embedded image of $G$ in $\operatorname{Sym}(p q)$. In this family, it was always the case that

$$
\mu(G(p, p, q))=\mu\left(G(p, p, q) \times C_{\mathrm{Sym}(p q)}(G(p, p, q))\right)=p q,
$$

and so examples of strict inequality in (1) was assured. The smallest minimal degree of a direct product in this family was 10 , furnished by taking $G$ to be $G(2,2,5)$ and thus $H=C_{\text {Sym }(10)}(G) \cong C_{2}$. In fact, one can take $G$ to be a split extension of the product of 4 copies of $C_{2}$ (a so-called deleted permutation module for $\operatorname{Sym}(5)$ over $\mathbb{F}_{2}$ ) by any subgroup of $\operatorname{Sym}(5)$ that contains a 5 -cycle (see [7] for a description of these complex reflection groups and exposition of the examples).

The main result of this paper is the following:

Theorem 1.1. If $G$ and $H$ are groups such that $\mu(G \times H) \leq 9$ then $\mu(G \times H)=\mu(G)+\mu(H)$.

\section{Background ANd Preliminaries}

Wright in [10] considered the class $\mathscr{C}$ of groups $G$ such that $\mu(G)=\mu\left(G_{1}\right)$ for some nilpotent subgroup $G_{1}$ of $G$. Wright noted (see Claim 1 and Claim 2 of [10]) that all symmetric, alternating and dihedral groups are members of $\mathscr{C}$. Because equality occurs in (1) whenever $G$ and $H$ are nilpotent (see [10, Corollary 2]), the following lemma is immediate and used often below without comment.

Lemma 2.1. If $G, H \in \mathscr{C}$ then $G \times H \in \mathscr{C}$ and $\mu(G \times H)=\mu(G)+\mu(H)$.

We now briefly state some background results that we will need during the course of our later proofs. Here, we follow the notation of [4], where further exposition and complete proofs can be found.

Definition 2.2. Let $G$ be a subgroup of $\operatorname{Sym}(A)$.

(i) We say that $G$ acts semi-regularly if, for all $x \in A, x^{g}=x$ implies $g=1$.

(ii) We say $G$ acts regularly if $G$ acts transitively and semi-regularly.

(iii) If $G$ acts transitively then a block for $G$ is a subset $B$ of $A$ such that, for all $g \in G$, $B^{g} \cap B=\varnothing$ or $B^{g}=B$.

(iv) If $H$ is a subgroup of $G$ then the set of fixed points of $H$ in $A$ is

$$
\operatorname{Fix}(H)=\left\{x \in A \mid x^{h}=x, \text { for all } h \in H\right\} .
$$

Theorem 2.3. Let $G$ be a transitive subgroup of $\operatorname{Sym}(A)$ and $H$ the stabiliser of a point in $A$. Then $C:=C_{\operatorname{Sym}(A)}(G) \cong N_{G}(H) / H$ and $C$ acts semi-regularly on $A$.

Corollary 2.4. Let $G$ be a subgroup of $\operatorname{Sym}(A)$ where $A=A_{1} \cup \ldots \cup A_{k}$ and the $A_{i}$ are the orbits of $G$, all of different sizes. Then

$$
C_{\operatorname{Sym}(A)}(G) \cong N_{G}\left(H_{1}\right) / H_{1} \times \ldots \times N_{G}\left(H_{k}\right) / H_{k}
$$

where $H_{i}$ is the stabiliser of a point in $A_{i}$ for $i=1, \ldots, k$.

Proof. Without loss of generality, we may suppose that $A_{i}=\left\{H_{i} g \mid g \in G\right\}$ for each $i$. The map that takes $x \in N_{G}\left(H_{i}\right)$ to the permutation $H_{i} g \mapsto H_{i} x^{-1} g$ for $g \in G$ induces an isomorphism

$$
\Phi_{i}: N_{G}\left(H_{i}\right) / H_{i} \longrightarrow C_{i}
$$


where $C_{i}:=C_{\operatorname{Sym}\left(A_{i}\right)}\left(G_{\mid A_{i}}\right)$. Gluing these maps together, we get an embedding

$$
\Phi=\left(\Phi_{1}, \ldots, \Phi_{k}\right): N_{G}\left(H_{1}\right) / H_{1} \times \ldots \times N_{G}\left(H_{k}\right) / H_{k} \longrightarrow C_{\operatorname{Sym}(A)}(G),
$$

where the images are juxtaposed in the usual way since the orbits are disjoint. We claim that $\Phi$ is onto.

Let $\theta$ be an arbitrary element of $C_{\operatorname{Sym}(A)}(G)$. Suppose first that, for some $i \neq j$, there exists an $x \in A_{i}$ such that $x \theta \in A_{j}$. Since $\theta$ centralises $G$, the restriction of $\theta$ to $A_{i}$ is an injective map into $A_{j}$, so that $\left|A_{i}\right| \leq\left|A_{j}\right|$. But $\theta^{-1}$ also centralises $G$, so similarly $\left|A_{j}\right| \leq$ $\left|A_{i}\right|$, whence $\left|A_{i}\right|=\left|A_{j}\right|$, contradicting that the orbits have different sizes. Hence the orbits of $\theta$ respect the partition of $A$ given by $A_{1}, \ldots, A_{k}$. For each $i=1, \ldots, k, \theta_{\mid A_{i}}: H_{i} \mapsto H_{i} g_{i}$, for some $g_{i} \in G$ and it quickly follows that $g_{i} \in N_{G}\left(H_{i}\right)$ and $\theta_{\mid A_{i}}=\left(H_{i} g_{i}^{-1}\right) \Phi_{i}$. Hence $\theta=\left(H_{1} g_{1}^{-1}, \ldots, H_{k} g_{k}^{-1}\right) \Phi$, completing the proof that $\Phi$ is onto.

The following propositions are well-known (see [4] or [3] for example).

Proposition 2.5. Let $G$ be a transitive subgroup of $\operatorname{Sym}(A)$ and $H$ the stabiliser of a point. Then $\operatorname{Fix}(H)$ is a block for $G$, the induced permutation group on the block $\operatorname{Fix}(H)$ is regular and $|\operatorname{Fix}(H)|=\left|N_{G}(H) / H\right|$.

Proposition 2.6. Let $G$ be a transitive subgroup of $\operatorname{Sym}(A)$ with non-trivial block B. Let $G_{B}^{B}$ denote the induced permutation group on the block $B$ and $\bar{G}$ denote the induced action on the set of blocks. Then $G$ embeds in the wreath product $G_{B}^{B} \imath \bar{G}$.

We list a few more technical observations here of a general nature relating to minimal embeddings which we will use repeatedly later on.

Lemma 2.7. Suppose $G$ is a subgroup of $\operatorname{Sym}\left(A_{1}\right) \times \ldots \times \operatorname{Sym}\left(A_{k}\right)$ such that $\mu(G)=$ $\left|A_{1}\right|+\ldots+\left|A_{k}\right|$.

(i) $\mu(G \pi)=\sum_{i \in X}\left|A_{i}\right|$ for any projection $\pi$ onto $\prod_{i \in X} \operatorname{Sym}\left(A_{i}\right)$ for $X$ any subset of $\{1, \ldots, k\}$.

(ii) For all $i$ in $\{1, \ldots, k\}$, there exists an $\alpha \neq 1$ such that $(1, \ldots, \alpha, \ldots, 1)$ is contained in $G$, where $\alpha$ is located in the $i$-th place.

(iii) If $\left|A_{1}\right|=2$, then $G \cong C_{2} \times H$ where $H$ is a subgroup of $\operatorname{Sym}\left(A_{2}\right) \times \ldots \times \operatorname{Sym}\left(A_{k}\right)$ and $\mu(H)=\left|A_{2}\right|+\ldots+\left|A_{k}\right|$.

(iv) If $\left|A_{1}\right|=3$, then $(\alpha, 1, \ldots, 1)$ is an element of $G$ for some 3 -cycle $\alpha$.

(v) If $\left|A_{1}\right|=4$ and $(\alpha, 1, \ldots, 1)$ is an element of $G$ for some 3 -cycle $\alpha$, then $G$ contains $\operatorname{Alt}\left(A_{1}\right) \times\{1\} \times \ldots \times\{1\}$.

(vi) If $\left|A_{1}\right|=4$, say $A_{1}=\{a, b, c, d\}$, and $((a b), 1, \ldots, 1) \in G$, then $((c d), 1, \ldots, 1) \in G$.

(vii) If $G \pi$ is transitive where $\pi$ is the projection onto the first coordinate and $\left|A_{1}\right|=p$ for some prime $p$ such that $p>\left|A_{i}\right|$ for all $i \in\{2, \ldots, k\}$, then $(\alpha, 1, \ldots, 1)$ is an element of $G$ for some p-cycle $\alpha$.

Proof. Put $n=\left|A_{1}\right|+\ldots+\left|A_{k}\right|$. For (i), we observe that if $\mu(G \pi)<\sum_{i \in X}\left|A_{i}\right|$, then pasting projections gives an embedding of $G$ in $\operatorname{Sym}(n-1)$, contradicting that $\mu(G)=n$.

For (ii), let $\pi$ be the projection onto $\prod_{j \neq i} \operatorname{Sym}\left(A_{j}\right)$. Then $\operatorname{ker}\left(\pi_{\mid G}\right)$ is non-trivial, for otherwise $G$ embeds inside $\operatorname{Sym}(n-1)$, again contradicting that $\mu(G)=n$. 
For (iii), suppose $\left|A_{1}\right|=2$. By (ii), $g=(\alpha, 1, \ldots, 1) \in G$, where $\operatorname{Sym}\left(A_{1}\right)=\{1, \alpha\}$. Let $K$ be the kernel of the projection of $G$ onto the first coordinate, so

$$
K \cap\langle g\rangle=\{(1, \ldots, 1)\} \quad \text { and } \quad G=\langle g\rangle K,
$$

so that $G$ is the internal direct product of $\langle g\rangle$ and $K$. But the first coordinate of each element of $K$ is 1 , so $K \cong H$, where $H$ is the result of ignoring the first coordinate. Thus $G \cong\langle g\rangle \times K \cong C_{2} \times H$. Clearly $H$ is a subgroup of $\operatorname{Sym}\left(A_{2}\right) \times \ldots \times \operatorname{Sym}\left(A_{k}\right)$ and $H=G \pi$ where $\pi$ projects onto $\operatorname{Sym}\left(A_{2}\right) \times \ldots \times \operatorname{Sym}\left(A_{k}\right)$, so that $\mu(H)=\left|A_{2}\right|+\ldots+\left|A_{k}\right|$, by (i).

For (iv), suppose $\left|A_{1}\right|=3$. By (ii), we have that $g=(\alpha, 1, \ldots, 1) \in G$ for some $\alpha \neq 1$ in $\operatorname{Sym}\left(A_{1}\right)$. If $\alpha$ is a 3 -cycle, then we are done so suppose $\alpha=(a b)$ where $A_{1}=\{a, b, c\}$. Since $\mu(G)=n$ there exists $h=\left(\beta, h_{2}, \ldots, h_{k}\right) \in G$ for some $\beta$ that moves $c$, that is, $\beta$ is an element of $\{(a c),(b c),(a b c),(a c b)\}$. It follows that $[\alpha, \beta]=(a b c)$ or $(a c b)$ and so $[g, h]=([\alpha, \beta], 1 \ldots, 1)$ is contained in $G$.

For (v) and (vi), suppose $\left|A_{1}\right|=4$, say $A_{1}=\{a, b, c, d\}$ and let $\pi$ denote projection onto the first coordinate. If $g=(\alpha, 1, \ldots, 1) \in G$ where $\alpha=(a b c)$ then, since $\mu(G)=$ $n$, there exists some $h=\left(\beta, h_{2}, \ldots, h_{n}\right) \in G$ such that $\beta$ moves $d$, and then $\left\langle g, g^{h}\right\rangle \cong$ $\operatorname{Alt}\left(A_{1}\right) \times\{1\} \times \ldots \times\{1\}$, verifying (v). For (vi), suppose that $g=(\alpha, 1, \ldots, 1) \in G$ where $\alpha=(a b)$. Suppose first that $G \pi$ is not transitive. Then, since $\mu(G)=n$, both $\{a, b\}$ and $\{c, d\}$ are orbits of $G \pi$. By part (ii) applied to $G$ identified with a subgroup of $\operatorname{Sym}(\{a, b\}) \times \operatorname{Sym}(\{c, d\}) \times \ldots \times \operatorname{Sym}\left(A_{k}\right)$, it follows that $((c d), 1, \ldots, 1) \in G$, under the original embedding in $\operatorname{Sym}\left(A_{1}\right) \times \ldots \times \operatorname{Sym}\left(A_{k}\right)$, and the conclusion of (vi) holds. Thus we may suppose $G \pi$ is transitive, so there exists some $h=\left(\beta, h_{2}, \ldots, h_{n}\right), h^{\prime}=\left(\gamma, h_{2}^{\prime}, \ldots, h_{n}^{\prime}\right) \in$ $G$ such that $\beta$ moves $a$ to $c$ and $\gamma$ moves $a$ to $d$. If $\alpha^{\beta}=(c d)$ or $\alpha^{\gamma}=(c d)$ then $g^{h}$ or $g^{h^{\prime}}=((c d), 1, \ldots, 1)$, and the conclusion of (vi) holds. Thus we may suppose that $\alpha^{\beta}=(c a)$ or $(c b)$ and $\alpha^{\gamma}=(d a)$ or $(d b)$. But $\alpha^{(c b)}=(c a)$ and $\alpha^{(d a)}=(d b)$, so in all cases we have $(c a)(d b) \in G \pi$. Hence there exists some $h^{\prime \prime}=\left((c a)(d b), h_{2}^{\prime \prime}, \ldots, h_{k}^{\prime \prime}\right) \in G$, so $((c d), 1, \ldots, 1)=g^{h^{\prime \prime}} \in G$, completing the proof of (vi).

For (vii), suppose $G \pi$ is transitive where $\pi$ is projection onto the first coordinate and $\left|A_{1}\right|=p$, where $p$ is a prime and $p>\left|A_{i}\right|$ for all $i \geq 2$. Then $G$ contains an element $\left(\alpha_{1}, \ldots, \alpha_{k}\right)$ of order $p$, since $p$ divides $|G|$. This implies that $\alpha_{2}=\ldots=\alpha_{k}=1$ since there is insufficient room for $p$-cycles in $\operatorname{Sym}\left(A_{2}\right), \ldots, \operatorname{Sym}\left(A_{k}\right)$.

Lemma 2.8. Let $G$ be a subgroup of $\operatorname{Sym}(n)$ such that $\mu(G)=n \geq 2$. Suppose that the stabiliser $H$ of some point is normal in $G$. Then $G / H$ is a cyclic group of prime power order, a Klein four-group or a generalised quaternion 2-group.

Proof. We may suppose that $H$ is the stabiliser of 1 and the orbit of $G$ containing 1 is $\{1, \ldots, m\}$ for some integer $m$ such that $2 \leq m \leq n$. Because $H$ is normal, $H$ stabilises each of $1, \ldots, m$. Put $K=\left.G\right|_{\{1, \ldots, m\}}$; the induced permutation group on $\{1, \ldots, m\}$. Then $K \cong G / H$ and $|K|=m$. By Lemma 2.7 (i), $\mu(K)=m$. Hence, by [6, Theorem 1], $K$ is a cyclic group of prime power order, a Klein four-group or a generalised quaternion 2-group.

Corollary 2.9. Let $G$ be a subgroup of $\operatorname{Sym}(n)$ such that $\mu(G)=n$ and $n \leq 9$. Then $C:=C_{\operatorname{Sym}(n)}(G)$ is abelian or isomorphic to $Q_{8}$. In particular $C \in \mathscr{C}$. 
Proof. We may assume $G$ is nontrivial, so each orbit contains at least two points. Consider any stabiliser $H$ of any point. Then $\left|N_{G}(H) / H\right|$ divides $|G: H|$ and $|G: H| \in\{2, \ldots, 9\}$. If $|G: H|=6$ then, by Lemma $2.8, H$ is not normal in $G$, so that $\left|N_{G}(H) / H\right| \in\{1,2,3\}$. It follows that, in all cases, $\left|N_{G}(H) / H\right| \in\{1,2,3,4,5,7,8,9\}$. (Thus, if $\left|N_{G}(H) / H\right| \neq 8$ then $N_{G}(H) / H$ is abelian.)

Suppose that $G$ is transitive. By Theorem 2.3, $C \cong N_{G}(H) / H$ where $H$ is the stabiliser of a point. If $H$ is normal in $G$ then $|G / H| \leq 9$ and, by Lemma $2.8, C \cong G / H$ is cyclic of prime power order, a Klein four-group or isomorphic to $Q_{8}$, and the corollary holds. If $H$ is not normal in $G$ then $|C|=\left|N_{G}(H) / H\right| \leq 4$, so certainly $C$ is abelian, and the corollary holds.

Suppose now that $G$ is intransitive. In cases where the orbit sizes are pairwise distinct, it follows from the first paragraph of this proof and from Corollary 2.4 that $C$ is a direct product of groups of order at most 7 , avoiding 6 , so is abelian. It remains to consider the following cases for orbit sizes: (i) $5,2,2$; (ii) 4, 4; (iii) 4,2, 2; (iv) $3,3,3$; (v) $3,2,2,2$; (vi) $3,3,2$; (vii) $3,2,2$; (viii) 3,3 ; (ix) $2,2,2,2$; (x) $2,2,2$; (xi) 2 , 2. In case (i), by Lemma 2.7 , $G$ contains a subgroup permutation equivalent to $K=\langle(12345),(67),(89)\rangle$, so that $C$ is isomorphic to a subgroup of $C_{\mathrm{Sym}(9)}(K) \cong K$, which is abelian. In cases (ii), (iii) and (ix), by Lemma $2.7, G$ contains a subgroup permutation equivalent to one of

$$
\begin{aligned}
& K_{1}=\langle(12),(34),(56),(78)\rangle ; \\
& K_{2}=\langle(12)(34),(13)(24),(56),(78)\rangle ; \\
& K_{3}=\langle(12)(34),(13)(24),(56)(78),(57)(68)\rangle ;
\end{aligned}
$$

so that $C$ is isomorphic to a subgroup of $C_{\mathrm{Sym}(8)}\left(K_{i}\right) \cong C_{2} \times C_{2} \times C_{2} \times C_{2}$, for some $i$, so $C$ is abelian. In case (iv), by Lemma $2.7, G$ contains a subgroup permutation equivalent to $K=\langle(123),(456),(789)\rangle$, so that $C$ is isomorphic to a subgroup of $C_{\operatorname{Sym}(9)}(K) \cong K$, which is abelian. The remaining cases are dealt with similarly, and the proof is complete.

Remark 2.10. In the above proof $C$ is abelian except in one instance, when $G \cong Q_{8}$, in which case $C \cong Q_{8}$. A realisation of this would be, for example, when $G$ and $C$ are as follows:

$$
\begin{aligned}
& G=\langle(1234)(5678),(1537)(2846)\rangle, \\
& C=\langle(1234)(5876),(1537)(2648)\rangle .
\end{aligned}
$$

The following simple observation will be used repeatedly in the sequel.

Proposition 2.11. Let $G \in \mathscr{C}$ with $\mu(G)=n$ and identify $G$ with its embedded image in $\operatorname{Sym}(n)$. Let $C:=C_{\operatorname{Sym}(n)}(G)$ be the centraliser of $G$ in $\operatorname{Sym}(n)$ with respect to this minimal embedding and suppose that $C$ is nilpotent. Then every nontrivial subgroup of $C$ intersects $G$ nontrivially.

Proof. Suppose for a contradiction that there is a nontrivial subgroup $P$ of $C$ such that $G \cap P=\{1\}$. Then $\langle G, P\rangle=G P$ is a subgroup of $\operatorname{Sym}(n)$ that is an internal direct product of $G$ and $P$. But $P$ is nilpotent, being a subgroup of a nilpotent group, so $P \in \mathscr{C}$. Since $G \in \mathscr{C}$ we have

$$
\mu(G P)=\mu(G \times P)=\mu(G)+\mu(P)>n,
$$


contradicting that $\mu(G P) \leq n$.

Corollary 2.12. With notation as in the previous proposition, if $C$ is elementary abelian, then $C \leq G$.

Proof. If $C$ is elementary abelian and $1 \neq c \in C$ then $\langle c\rangle$ is a subgroup of order $p$, so $c \in G$ by the previous proposition.

\section{Case by Case that 10 is Minimal}

In this section we will prove Theorem 1.1 below in stages, so that there are no examples of strict inequality in (1) in $\operatorname{Sym}(n)$ for $n \leq 9$. This is clear for $n=2$ and $n=3$. Our approach for $n=4, \ldots, 9$ is to show that, for a minimally embedded group $G$ in $\operatorname{Sym}(n)$, there is no nontrivial subgroup of the centraliser of $G$ in $\operatorname{Sym}(n)$ that intersects trivially with $G$. For the most part, this will follow by applying Proposition 2.11, revealing the pervasiveness of Wright's class $\mathscr{C}$ for permutation groups of small degree.

\subsection{The $\operatorname{Sym}(4), \operatorname{Sym}(5)$ and $\operatorname{Sym}(6)$ Cases.}

Proposition 3.1. Let $G$ be a finite group such that $\mu(G) \leq 6$. Then $G \in \mathscr{C}$.

Proof. First suppose that $\mu(G)=4$. If $G$ acts intransitively with respect to the embedding in $\operatorname{Sym}(4)$, then $G \cong C_{2} \times C_{2}$ by Lemma 2.7 (iii), so $G \in \mathscr{C}$. Suppose that $G$ acts transitively. Then $G$ has a core-free subgroup $H$ of index 4, so that the Sylow 2-subgroups of $G$ have size 4 or 8 . Hence, a copy of $C_{4}$ or $C_{2} \times C_{2}$ is a subgroup of $G$. Both of these are nilpotent and have minimal degree 4 , so again $G \in \mathscr{C}$.

Now suppose that $\mu(G)=5$. If $G$ acts transitively, then $G$ contains a subgroup of index 5 and so contains a copy of $C_{5}$, implying that $G \in \mathscr{C}$. Suppose that $G$ acts intransitively. By minimality, the action of $G$ must have two orbits, of sizes 2 and 3 respectively. By Lemma 2.7 (iii),(iv), $G$ contains a subgroup isomorphic to $C_{3} \times C_{2}$, so again $G \in \mathscr{C}$.

Finally suppose that $\mu(G)=6$. We may identify $G$ with its embedded image in $\operatorname{Sym}(6)$. If 9 or 16 divides $|G|$, then $G$ contains a Sylow 2 or 3-subgroup of $\operatorname{Sym}(6)$ and hence a copy of $C_{3} \times C_{3}$ or $C_{2} \times C_{2} \times C_{2}$, so that $G \in \mathscr{C}$.

We may suppose therefore that neither 9 or 16 divides $|G|$. Suppose first that $G$ acts intransitively. It follows by Lemma 2.7 (iii), (iv), that $G$ contains a subgroup that is an internal direct product of subgroups $K$ and $L$ of minimal degrees less than 6 but adding up to 6. By previous cases, $K, L \in \mathscr{C}$, so $K \times L \in \mathscr{C}$, and it follows that $G \in \mathscr{C}$.

Henceforth we may suppose that $G$ acts transitively. In particular, $G$ contains a subgroup of index 6 , so that the possible orders of $G$ are $6,12,24,30,60$ or 120 . If $|G|=6$ or 12 then, in the latter case from the Appendix, $\mu(G) \neq 6$, which is impossible. If $|G|=24$ then, from the Appendix, either $\mu(G) \neq 6$, which is impossible, or $G$ contains a copy of $C_{2} \times C_{2} \times C_{2}$, so that $G \in \mathscr{C}$. If $|G|=30$ then $G$ contains a copy of $C_{5} \times C_{3}$ so that $\mu(G) \geq 8$, which is again impossible.

Henceforth we may suppose that $|G|=60$ or 120 . It is well known that the only subgroups of $\operatorname{Sym}(6)$ of orders 60 and 120 are isomorphic to Alt(5) and Sym(5) respectively, so that $\mu(G)=5$, which is impossible. This completes the proof of the proposition. 
Corollary 3.2. Let $G$ be a transitive subgroup of $\operatorname{Sym}(6)$ such that $\mu(G)=6$. Then $C:=C_{\operatorname{Sym}(6)}(G) \leq G$.

Proof. Let $H$ be the stabiliser of a point, so $|G: H|=6$. By Theorem 2.3, $C \cong N_{G}(H) / H$. By Lemma $2.8, H$ is not normal in $G$, so $|C|=1,2$ or 3 . By Proposition $3.1, G \in \mathscr{C}$, so, by Proposition 2.11, $C \leq G$.

3.2. The $\operatorname{Sym}(7)$ Case. Throughout this subsection, put

$$
H:=\langle(123),(12)(4567)\rangle \cong\left\langle a, b \mid a^{3}=b^{4}=1, a^{b}=a^{-1}\right\rangle .
$$

Proposition 3.3. Let $H$ be as in (2). Then $\mu(H)=7, H \notin \mathscr{C}$ and $H$ is up to isomorphism the unique proper subdirect product of $\operatorname{Sym}(3) \times C_{4}$. Further $C_{\operatorname{Sym}(7)}(H)=\langle(4567)\rangle$.

Proof. It is easily verified that $\mu(H)=7$ (and minimal degrees of groups of order 12 are listed in the Appendix) and that the nilpotent subgroups of $G$ are isomorphic to $C_{2}, C_{3}, C_{4}$ and $C_{2} \times C_{3}$, all of which have minimal degree strictly less than 7 . Hence $G \notin \mathscr{C}$. It is easy to check that $\operatorname{Sym}(3) \times C_{4}$ has a unique subdirect product of order 12 containing an element of order 4 , which must therefore be isomorphic to $H$.

Put $z=(4567)$. Clearly $\langle z\rangle \subseteq C:=C_{\operatorname{Sym}(7)}(H)$. Note that the orbits of $H$ are $\{1,2,3\}$ and $\{4,5,6,7\}$ and are of different sizes. By Corollary 2.4, $C \cong N_{H}\left(H_{3}\right) / H_{3} \times N_{H}\left(H_{4}\right) / H_{4}$, where $H_{3}=\langle(12)(4567)\rangle$ and $H_{4}=\langle(123)\rangle$ are the stabilisers of 3 and 4 respectively. But $N_{H}\left(H_{3}\right)=H_{3}$ and $N_{H}\left(H_{4}\right)=H$, so that $C \cong H / H_{4} \cong C_{4}$. Therefore $C=\langle z\rangle$.

This group $H$ is also unique in the following sense.

Theorem 3.4. Let $G$ be a group such that $\mu(G)=7$ and $G \notin \mathscr{C}$. Then the image of any minimal embedding of $G$ in $\operatorname{Sym}(7)$ is permutation equivalent to $H$. In particular, $G \cong H$.

Proof. We may regard $G$ as a subgroup of $\operatorname{Sym}(7)$. If $G$ is transitive then 7 divides $|G|$, being the index of a point stabiliser, so that $G$ contains a copy of $C_{7}$, and $\mu(G)=7=\mu\left(C_{7}\right)$, contradicting that $G \notin \mathscr{C}$. Hence $G$ is intransitive.

If $G$ has an orbit of size 2, then by Lemma 2.7 (iii), $G \cong C_{2} \times K$ for some group $K$ such that $\mu(K)=5$, so $K \in \mathscr{C}$, by Proposition 3.1, whence $G \in \mathscr{C}$, a contradiction. It follows that $G$ has one orbit of size 3 and one of size 4 . Without loss of generality we may suppose these orbits are $X_{1}=\{1,2,3\}$ and $X_{2}=\{4,5,6,7\}$. By parts (ii) and (iv) of Lemma 2.7, we may, without loss of generality, assume that $\alpha:=(123) \in G$ and there exists $\beta \in G$ such that $\beta$ fixes $X_{1}$ pointwise and moves a letter from $X_{2}$.

If $\beta$ is a 4 -cycle then $G$ contains the subgroup $\langle\alpha, \beta\rangle \cong C_{3} \times C_{4}$. If $\beta$ is a 3 -cycle or a 2-cycle, then, by Lemma 2.7 (v) and (vi), $G$ contains a subgroup isomorpic to $C_{3} \times \operatorname{Alt}(4)$ or $C_{3} \times C_{2} \times C_{2}$ respectively. In each of these cases, $G \in \mathscr{C}$, leading to a contradiction.

Hence $\beta$ must be a product of two disjoint 2-cycles. Without loss of generality, we suppose that $\beta=(46)(57)$. For any $\gamma \in G$, we will write $\gamma_{1}=\left.\gamma\right|_{X_{1}}$ and $\gamma_{2}=\left.\gamma\right|_{X_{2}}$, so that $\gamma=\gamma_{1} \gamma_{2}$.

Let $\pi$ be projection onto $\operatorname{Sym}\left(X_{2}\right)$, so that $G \pi$ must be a transitive $\operatorname{subgroup}$ of $\operatorname{Sym}\left(X_{2}\right)$. By [4, Table 2.1] the transitive subgroups of $\operatorname{Sym}\left(X_{2}\right)$ are itself, $\operatorname{Alt}\left(X_{2}\right)$ or isomorphic to $C_{2} \times C_{2}, D_{8}$ or $C_{4}$. 
Case (i): $G \pi=\operatorname{Sym}\left(X_{2}\right)$ or $\operatorname{Alt}\left(X_{2}\right)$.

There is some $\gamma \in G$ such that $\gamma_{2}=(456)$, with $\gamma_{1}$ being a 2-cycle or a power of $\alpha$. It readily follows that $\gamma_{2} \in G$. By Lemma 2.7 (v), $C_{3} \times \operatorname{Alt}(4)$ is isomorphic to a subgroup of $G$, and it follows that $G \in \mathscr{C}$, a contradiction.

Case (ii): $G \pi=\langle(45)(67),(46)(57)\rangle$.

There is some $\gamma \in G$ such that $\gamma_{2}=(45)(67)$. If $\gamma_{1} \in\langle\alpha\rangle$ then $\gamma_{2} \in G$ and $\left\langle\alpha, \beta, \gamma_{2}\right\rangle$ is a subgroup of $G$ isomorphic to $C_{3} \times C_{2} \times C_{2}$, so that $G \in \mathscr{C}$, a contradiction. Hence $\gamma_{1} \notin\langle\alpha\rangle$, so, without loss of generality, $\gamma_{1}=(12)$. Then $\langle\alpha, \beta, \gamma\rangle$ is a subgroup of $G$ isomorphic to $\operatorname{Sym}(3) \times C_{2}$, of minimal degree 5 , so cannot exhaust all of $G$. Hence there exists some $\delta \in G \backslash\langle\alpha, \beta, \gamma\rangle$. If $\delta_{2}=1$ or $\beta$, then $\delta_{1} \in G$ and $\delta_{1}$ is a 2 -cycle, so that $\langle\alpha, \beta, \gamma, \delta\rangle \cong \operatorname{Sym}(3) \times C_{2} \times C_{2}$, so that $G \in \mathscr{C}$, a contradiction. Hence $\delta_{2}=(45)(67)$ or (47)(56) and $\delta_{1} \in\langle\alpha\rangle$. Then $\langle\alpha, \beta, \delta\rangle \cong C_{3} \times C_{2} \times C_{2}$, so that $G \in \mathscr{C}$, a contradiction.

Case (iii): $G \pi \cong D_{8}$.

Either $\beta$ is central or $\beta$ inverts a 4-cycle in $G \pi$. Suppose first that $\beta$ is central in $G \pi$. Then there are some $\gamma, \delta \in G$ such that $\gamma_{2}=(4567)$ and $\delta_{2}=(46)$. If $\gamma_{1} \in\langle\alpha\rangle$ or $\delta_{1} \in\langle\alpha\rangle$ then $\langle\alpha, \gamma\rangle \cong C_{3} \times C_{4}$ or $\langle\alpha, \beta, \delta\rangle \cong C_{3} \times C_{2} \times C_{2}$ respectively, so that $G \in \mathscr{C}$, a contradiction. Hence $\gamma_{1}$ and $\delta_{1}$ are both 2-cycles. By conjugating $\delta$ by a power of $\alpha$, without any loss of generality, we may assume $\gamma_{1}=\delta_{1}$. But then $\gamma \delta=(45)(67)$, so that $\langle\alpha, \beta, \gamma \delta\rangle \cong C_{3} \times C_{2} \times C_{2}$, so that $G \in \mathscr{C}$, a contradiction. Hence $\beta$ inverts a 4-cycle in $G \pi$, so there is some $\varepsilon \in G$ such that $\varepsilon_{2}$ is a 4 -cycle and $\varepsilon_{2}^{2} \neq \beta$. If $\varepsilon_{1} \in\langle\alpha\rangle$ then $\langle\alpha, \varepsilon\rangle \cong C_{3} \times C_{4}$, so that $G \in \mathscr{C}$, a contradiction. Hence $\varepsilon_{1}$ is a 2-cycle, so that $\left\langle\alpha, \beta, \varepsilon^{2}\right\rangle \cong C_{3} \times C_{2} \times C_{2}$, so that $G \in \mathscr{C}$, a contradiction.

Cases (i), (ii) and (iii) produce contradictions, so we must have $G \pi \cong C_{4}$. Hence there is some $\gamma \in G$ such that $\gamma_{2}=(4567)$. If $\gamma_{1} \in\langle\alpha\rangle$ then $\langle\alpha, \gamma\rangle \cong C_{3} \times C_{4}$, so that $G \in \mathscr{C}$, a contradiction. Hence $\gamma_{1}$ is a 2-cycle, and it follows that $(12)(4567) \in G$, so that $H \leq G$. If $H \neq G$ then $G \cong \operatorname{Sym}(3) \times C_{4}$, so that $G \in \mathscr{C}$, a contradiction. Hence $H=G$, and the theorem is proved.

3.3. The $\operatorname{Sym}(8)$ Case. In this section we prove that all intransitive subgroups and all but two transitive subgroups of $\operatorname{Sym}(8)$ of minimal degree 8 are members of $\mathscr{C}$. The two exceptions up to isomorphism that are not members of $\mathscr{C}$ (see Theorem 3.6 below) turn out to be primitive:

$$
\begin{aligned}
K & =\langle(12)(34)(56)(78),(13)(24)(57)(68),(15)(26)(37)(48),(2354786)\rangle \\
& \cong\left(C_{2} \times C_{2} \times C_{2}\right) \rtimes C_{7}, \\
L & =K\langle(357)(468)\rangle \cong\left(C_{2} \times C_{2} \times C_{2}\right) \rtimes\left(C_{7} \rtimes C_{3}\right) .
\end{aligned}
$$

That these groups are not in $\mathscr{C}$ can be seen by inspection: all nilpotent subgroups of $K$ and $L$ have minimal degree less than 8. Also note (for the proof of Theorem 3.7 below) that both $C_{\mathrm{Sym}(8)}(K)$ and $C_{\mathrm{Sym}(8)}(L)$ are trivial.

Proposition 3.5. Let $G$ be a group such that $\mu(G)=8$ and the minimal faithful representation of $G$ is intransitive. Then $G \in \mathscr{C}$. 
Proof. We may suppose throughout that $G$ is not a 2-group. If $G$ has an orbit of size 2 or 3 then, by Lemma 2.7, $G$ contains a subgroup that is an internal direct product of subgroups $H_{1}$ and $H_{2}$ of minimal degrees less than 7 but adding up to 8 , so, by Proposition $3.1, H_{1}$ and $H_{2}$ both lie in $\mathscr{C}$, whence $G \in \mathscr{C}$.

Hence we may suppose that $G$ has exactly two orbits of size 4 , which we may take to be $\{1,2,3,4\}$ and $\{5,6,7,8\}$. In particular, $|G|$ must be divisible by 3 and 4 , but not by 5 or 7. Let $S$ denote a Sylow 2 -subgroup and $T$ a Sylow 3 -subgroup of $G$. If 32 divides $|S|$, then $\mu(S) \geq 8$ (because 32 does not divide 7 !), so that $\mu(S)=8$ and $G \in \mathscr{C}$. If 9 divides $|G|$, then $T$ is a Sylow 3-subgroup of $\operatorname{Sym}(8)$, so, without loss of generality, $T=\langle(123),(567)\rangle$ and, by Lemma $2.7(\mathrm{v}), G$ contains the subgroup $\operatorname{Alt}(\{1,2,3,4\}) \times \operatorname{Alt}(\{5,6,7,8\})$, so $G \in \mathscr{C}$.

Henceforth we may suppose that 32 and 9 do not divide $|G|$, so that $|G|=12,24$, or 48. From the Appendix, the only possibilities for $G$ up to isomorphism are $C_{2} \times C_{2} \times \mathrm{Alt}_{4}$, $\left(C_{2} \times C_{2} \times C_{2} \times C_{2}\right) \rtimes C_{3}, C_{4} \times \operatorname{Alt}(4)$ and $\operatorname{Alt}(4) \rtimes C_{4}$ (we exclude $S L(2,3), G L(2,3)$ as they act transitively). For the first three cases, $G$ can clearly be seen to lie in $\mathscr{C}$. In the last case, $G$ contains a copy of the semidirect product $\left(C_{4} \times C_{2}\right) \rtimes C_{2}$ that has minimal degree 8 , so $G \in \mathscr{C}$.

Theorem 3.6. Let $G$ be a group such that $\mu(G)=8$ and the minimal permutation representation is transitive. Then $G \in \mathscr{C}$ or $G$ is permutation isomorphic to $K \cong\left(C_{2} \times C_{2} \times C_{2}\right) \rtimes C_{7}$ or $L \cong\left(C_{2} \times C_{2} \times C_{2}\right) \rtimes\left(C_{7} \rtimes C_{3}\right)$.

Proof. Again we may suppose that $G$ is not a 2 -group and that 32 does not divide $|G|$. Since the representation is transitive, $G$ contains a core-free subgroup of index 8. In particular, 8 divides $|G|$. From Butler and McKay's 1983 classification of small transitive groups (see [2]), the order of $G$ must be $24,48,56,168$ or 336 , and their catalogue includes explicit descriptions of the structure of each possibility for $G$ up to permutation isomorphism. If $|G|=24$ or 48 then, as in the proof of Proposition 3.5, $G \in \mathscr{C}$. If $|G|=56$ then $G$ is permutation isomorphic to $K$. If $|G|=168$ then $G$ is permutation isomorphic to $L$ or to $\operatorname{PSL}(2,7)$. However, the latter possibility is ruled out because $\operatorname{PSL}(2,7)$ is simple with a subgroup of index 7, so embeds in $\operatorname{Sym}(7)$. If $|G|=336$ then $G$ is isomorphic to $\operatorname{PGL}(2,7)$, so has Sylow 2-subgroups isomorphic to $D_{16}$, which has minimal degree 8 , so that $G \in \mathscr{C}$, and the proof of the theorem is complete.

Combining results so far we can prove the following stepping-stone towards our main theorem (Theorem 1.1, also reproduced below as Theorem 3.13).

Theorem 3.7. If $G$ and $H$ are groups such that $\mu(G \times H) \leq 8$ then $\mu(G \times H)=\mu(G)+\mu(H)$.

Proof. Suppose by way of contradiction, that there exist subgroups $G$ and $H$ of $\operatorname{Sym}(8)$ such that $\langle G, H\rangle=G H$ is an internal direct product and $\mu(G \times H)<\mu(G)+\mu(H)$. Certainly $G$ and $H$ are nontrivial. By Lemma 2.1, it is not the case that both $G$ and $H$ lie in $\mathscr{C}$. Without loss of generality, we may suppose that $G \notin \mathscr{C}$. By Proposition $3.1, \mu(G) \geq 7$. If $\mu(G)=8$ then, by Proposition 3.5 and Theorem 3.6, $G$ is permutation isomorphic to $K$ or $L$, described in the preamble before Proposition 3.5, so $H$ is trivial (since $C_{\text {Sym }(8)}(K)$ and $C_{\text {Sym(8) }}(L)$ are both trivial), a contradiction. Hence $\mu(G)=7$.

By Theorem 3.4, $G \cong\left\langle a, b \mid a^{3}=b^{4}=1, a^{b}=a^{-1}\right\rangle$. Without loss of generality, we may take $a=(123)$ or $(123)(456)$ and $b$ a permutation of order 4 that inverts $a$ by conjugation. 
By a straightforward calculation, the only permutations of $\operatorname{Sym}(8)$ that invert (123)(456) by conjugation have order 2 or 6 , contradicting that $|b|=4$. Hence $a=(123)$. Clearly $b$ must be one of $\{(12) \sigma,(13) \sigma$ or $(23) \sigma\}$, where $\sigma$ is a 4-cycle that fixes 1,2 and 3 . Without loss of generality, $b=(13)(4567)$ and $G=\langle a, b\rangle$. Clearly $C_{\operatorname{Sym}(8)}(G)=\langle(4567)\rangle$ and $C_{\text {Sym(8) }}(G) \cap G=\langle(46)(57)\rangle$. But $\langle G, H\rangle=G H$ is an internal direct product, so $H \cap G=$ $\{1\}$ and $H \leq C_{\operatorname{Sym}(8)}(G)$. It follows quickly that $H$ is trivial, again a contradiction.

3.4. The Sym(9) Case. Again we consider in turn transitive and intransitive embeddings, though in both cases now there are groups that fall outside Wright's class $\mathscr{C}$. We show directly that every nontrivial subgroup of the centraliser intersects nontrivially with our minimally embedded group.

Proposition 3.8. Let $G$ be a group such that $\mu(G)=9$ and its minimally embedded image in $\operatorname{Sym}(9)$ is intransitive. Identify $G$ with its embedded image and let $C:=C_{\operatorname{Sym}(9)}(G)$. Then every nontrivial subgroup of $C$ intersects $G$ nontrivially.

Proof. If at any stage we conclude $G \in \mathscr{C}$ then we are done by Corollary 2.9 and Proposition 2.11. Without loss of generality, we only need to consider the following three cases.

Case (a): $G$ has an orbit $\{8,9\}$.

By Lemma $2.7, G \cong C_{2} \times H$ where $H \leq \operatorname{Sym}(\{1, \ldots, 7\})$ and $\mu(H)=7$. If $H \in \mathscr{C}$, then $G \in \mathscr{C}$, and we are done. Otherwise, by Theorem 3.4, without loss of generality, we may suppose that

$$
G=\langle(123),(13)(4567),(89)\rangle .
$$

Then $C=\langle(4567),(89)\rangle$ and $G \cap C=\langle(46)(57),(89)\rangle$. It quickly follows that every non-trivial subgroup of $C$ intersects non-trivially with $G$.

Case (b): $G$ has an orbit $\{7,8,9\}$ and no orbit of size two.

Subcase (i): $G$ has two other orbits both of size 3 .

By Lemma 2.7 (iv), $G$ contains a copy of $C_{3} \times C_{3} \times C_{3}$, so $G \in \mathscr{C}$, and we are done.

Subcase (ii): $G$ has an orbit $\{1, \ldots, 6\}$.

We may regard $G$ as a subgroup of $\operatorname{Sym}(\{1, \ldots, 6\}) \times \operatorname{Sym}(\{7,8,9\})$. Let $\pi_{1}$ and $\pi_{2}$ be projections onto $\operatorname{Sym}(\{1, \ldots, 6\})$ and $\operatorname{Sym}(\{7,8,9\})$ respectively. Let $K_{1}=\left.\operatorname{ker} \pi_{1}\right|_{G}$ and $K_{2}=\left.\operatorname{ker} \pi_{2}\right|_{G}$ and observe that $\left\langle K_{1}, K_{2}\right\rangle=K_{1} K_{2}$ is an internal direct product. By Lemma 2.7 (iv), we have $(789) \in G$. If moreover we have $G \pi_{2}=\langle(789)\rangle$ or that $G$ contains a 2-cycle supported only on $\{7,8,9\}$, then $G \cong H \times K$ where $\mu(H)=6$ and $\mu(K)=3$, so that $H, K \in \mathscr{C}$, by Proposition 3.1, whence $G \in \mathscr{C}$, and we are done. Hence we may assume that $G \pi_{2}=\operatorname{Sym}(\{7,8,9\})$ and that $G$ does not contain any 2-cycle supported only on $\{7,8,9\}$. Therefore $C$ may be identified with a subgroup of $\operatorname{Sym}(\{1, \ldots, 6\})$ and $C=C_{\operatorname{Sym}(6)}\left(G \pi_{1}\right)$. Let $H_{1}$ be the stabiliser of the letter 1 so that $H_{1} \pi_{1}$ has index 6 in $G \pi_{1}$. By Theorem 2.3 and Proposition 2.5, $|C|=\left|N_{G \pi_{1}}\left(H_{1} \pi_{1}\right) / H_{1} \pi_{1}\right|=\left|\operatorname{Fix}\left(H_{1} \pi_{1}\right)\right|=1,2,3$ or 6 . If $C$ is trivial then we are done. If $|C|=6$ then $H_{1} \leq K_{1}$, so that $H_{1}=K_{1}=\langle(789)\rangle$ and $|G|=18$, so that, from the Appendix, $G \cong D_{18}$, whence $G \in \mathscr{C}$, and we are done. We may suppose therefore 
that $|C|=2$ or 3. By Proposition 2.5 and Proposition 2.6, $G \pi_{1}$ embeds in $C_{3}$ 2 $\operatorname{Sym}(2)$ or $C_{2}$ 乙 $\operatorname{Sym}(3)$ as a transitive subgroup.

Suppose first that $G \pi_{1}$ embeds inside $C_{3}$ 乙 $\operatorname{Sym}(2)$. By Lemma 2.7 (i), $\mu\left(G \pi_{1}\right)=6$. By the classification of transitive subgroups of $\operatorname{Sym}(6)$ (see [4, Table 2.1]), it follows that 9 divides $\left|G \pi_{1}\right|$. Therefore 27 divides $|G|$ and $G$ contains a Sylow 3-subgroup with minimal degree 9 . Hence $G \in \mathscr{C}$, and we are done.

Now suppose that $G \pi_{1}$ embeds inside

$$
C_{2} \prec \operatorname{Sym}(3) \cong C_{2} \times \operatorname{Sym}(4) .
$$

Then $C$ has order 2 and may be identified under this isomorphism with the factor $C_{2}$ in the second group. Let $z$ be the generator of $C$; we will show that $z \in G$. Since $C=C_{\operatorname{Sym}(6)}\left(G \pi_{1}\right)$ from above, we have $C \leq G \pi_{1}$ by Corollary 3.2. Hence there is some $\sigma \in \operatorname{Sym}(\{7,8,9\})$ such that $\gamma:=z \sigma \in G$. We will show that $\sigma$ has order 1 or 3. Suppose to the contrary that $\sigma$ has order 2 . Since $G \pi_{2} \cong \operatorname{Sym}(3)$, we have $|G|=6\left|K_{2}\right|$. On the other hand, since no 2-cycle supported only on $\{7,8,9\}$ is contained in $G$, we have $K_{1}=\langle(789)\rangle \cong C_{3}$ and so $|G|=3\left|G \pi_{1}\right|$. Therefore, $\left|G \pi_{1}\right|=2\left|K_{2}\right|$, and so $|G|=2\left|K_{1}\right|\left|K_{2}\right|$. Observe that $\gamma \notin K_{1} K_{2}$ and $\gamma$ centralises $K_{2}$ and normalises $K_{1}$. Upon comparing orders,

$$
G=\left\langle K_{1}, K_{2}, \gamma\right\rangle=K_{1} K_{2}\langle\gamma\rangle \cong K_{2} \times\left(K_{1} \rtimes\langle\gamma\rangle\right) .
$$

Since $K_{1}=\langle(789)\rangle$ and $(789)^{\gamma}=(798)$, we have $K_{1} \rtimes\langle\gamma\rangle \cong \operatorname{Sym}(3)$, and since $K_{2}$ is isomorphic to a subgroup of $\operatorname{Sym}(4)$, by (3), $G$ is isomorphic to a $\operatorname{subgroup}$ of $\operatorname{Sym}(3) \times \operatorname{Sym}(4)$. Therefore $\mu(G) \leq \mu(\operatorname{Sym}(4) \times \operatorname{Sym}(3))=7$, contradicting that $\mu(G)=9$. Hence $\sigma$ has order 1 or 3 , and it follows immediately that $z \in G$. Hence $C \leq G$.

Case (c): $G$ has orbits $\{1,2,3,4\}$ and $\{5,6,7,8,9\}$.

Let $\pi_{1}$ and $\pi_{2}$ be projections onto $\operatorname{Sym}(\{1,2,3,4\})$ and $\operatorname{Sym}(\{5, \ldots, 9\})$ respectively. As before, let $K_{1}=\left.\operatorname{ker} \pi_{1}\right|_{G}$ and $K_{2}=\left.\operatorname{ker} \pi_{2}\right|_{G}$. By Lemma 2.7 (vii), without loss of generality, there is some $\gamma:=(56789) \in G$. If $G \pi_{2}=\langle\gamma\rangle$, then $G \cong G \pi_{1} \times C_{5}$ and $\mu\left(G \pi_{1}\right)=4$ by Lemma 2.7 (i), so that $G \pi_{1} \in \mathscr{C}$ by Proposition 3.1, whence $G \in \mathscr{C}$, and we are done. Therefore, $G \pi_{2}$ strictly contains $\langle\gamma\rangle$ and it follows that $C \leq \operatorname{Sym}(\{1,2,3,4\})$. If $G \pi_{1}=\operatorname{Alt}(\{1,2,3,4\})$ or $\operatorname{Sym}(\{1,2,3,4\})$, then $C=\{1\}$, and we are done. Therefore we may assume that $G \pi_{1}$ is isomorphic to $C_{4}, C_{2} \times C_{2}$ or $D_{8}$.

Suppose first that $G \pi_{1} \cong C_{4}$. Without loss of generality, $G \pi_{1}$ is generated by (1234), so that $C=G \pi_{1}$. By Lemma 2.7 (ii), (13)(24) $\in G$, and so $G$ meets every non-trivial subgroup of $C$ non-trivially.

Now suppose that $G \pi_{1} \cong C_{2} \times C_{2}$, so that $G \pi_{1}=C=\langle(12)(34),(13)(24)\rangle$. By Lemma 2.7 , we may suppose, without loss of generality, that $\alpha:=(12)(34) \in G$. We claim that $\beta:=(13)(24) \in G$. Certainly $\beta \sigma \in G$ for some $\sigma \in \operatorname{Sym}(\{5, \ldots, 9\})$ and if $\sigma \in G$ or $|\sigma|$ is coprime with $|\beta|$, then $\beta \in G$ and we are done. If $|\sigma|=6$ then we may replace $\sigma$ by $\sigma^{3}$. Thus we may suppose that $|\sigma|=2$ or 4 and that $\sigma \notin G$. Observe that $K_{2}=\langle\alpha\rangle$. On the one hand, $|G|=\left|K_{2}\right|\left|G \pi_{2}\right|=2\left|G \pi_{2}\right|$, and on the other, $|G|=\left|K_{1}\right|\left|G \pi_{1}\right|=4\left|K_{1}\right|$. Therefore $|G|=2\left|K_{1}\right|\left|K_{2}\right|$. Observe that $\left\langle K_{1}, K_{2}\right\rangle=K_{1} K_{2}$ is an internal direct product, $\beta \sigma \notin K_{1} K_{2}$ and $\beta \sigma$ centralises $K_{2}$ and normalises $K_{1}$. Therefore, comparing orders, we have

$$
G=\left\langle\beta \sigma, K_{1}, K_{2}\right\rangle=K_{1} K_{2}\langle\beta \sigma\rangle \cong K_{2} \times\left(K_{1}\langle\beta \sigma\rangle\right) \cong K_{2} \times\left(K_{1}\langle\sigma\rangle\right) .
$$


But $K_{2} \cong C_{2}$ and $K_{1}\langle\sigma\rangle$ is a subgroup of $\operatorname{Sym}(\{5, \ldots, 9\})$. Hence $G$ embeds in $C_{2} \times \operatorname{Sym}(5)$, so $\mu(G) \leq 7$, contradicting that $\mu(G)=9$, and we are done.

Finally suppose that $G \pi_{1} \cong D_{8}$. Without loss of generality, $G \pi_{1}=\langle r, s\rangle$ and $C=\left\langle r^{2}\right\rangle$ where $r:=(1234)$ and $s:=(12)(34)$. We claim that $r^{2} \in G$. This is immediate if $r \in G$, so we suppose that $r \notin G$. Certainly, $r \sigma \in G$ for some $\sigma \in \operatorname{Sym}(\{5, \ldots, 9\})$ such that $\sigma \notin G$ and $|\sigma|$ is divisible by 2. If $|\sigma|=2$ or 6 then $r^{2}=(r \sigma)^{|\sigma|} \in G$ and we are done. Thus, we may suppose $|\sigma|=4$. By Lemma 2.7 (ii), since $r, r^{-1} \notin G$, we have $r^{2}$ or $r^{j} s \in G$ for some $j$. In the first case we are done, and in the second case

$$
\sigma^{2}=\left(r^{j-1} s \sigma\right)^{2}=\left(r^{j} s r \sigma\right)^{2} \in G,
$$

so that $r^{2}=(r \sigma)^{2} \sigma^{2} \in G$, and we are done.

Remark 3.9. It can be verified by Magma that the only groups minimally embedded intransitively in $\operatorname{Sym}(9)$ that are not contained in $\mathscr{C}$ have orbits of size 2 and 7 , or orbits of size 4 and 5 . We do not prove this here as we do not need it for our main theorem. For more details the reader is referred to [8].

We have shown that if $G$ is a minimally embedded intransitive subgroup of $\operatorname{Sym}(9)$, then there is no subgroup $H$ of $\operatorname{Sym}(9)$ that centralises $G$ such that $\mu(G \times H)<\mu(G)+\mu(H)$. Before we deal with the transitive case we observe the following lemma, whose proof is a straightforward direct calculation.

Lemma 3.10. Let $W=C_{3} 2 \operatorname{Sym}(3)$ and let $x_{1}, x_{2}, x_{3}$ be the standard generators for the base group $B$. Let $U=\left\{x_{1}^{i} x_{2}^{j} x_{3}^{k} \in B \mid i+j+k \equiv 0 \bmod 3\right\}$ and $V=\left\langle x_{1} x_{2} x_{3}\right\rangle$. Then $V \subset U$ and $U$ and $V$ are the only non-trivial normal subgroups of $W$ strictly contained in $B$.

Proposition 3.11. Let $G$ be a group such that $\mu(G)=9$ and its minimally embedded image in $\operatorname{Sym}(9)$ is transitive. Identify $G$ with its image and put $C:=C_{\operatorname{Sym}(9)}(G)$. Then $C \leq G$.

Proof. We may assume $C$ is nontrivial and also that that $G$ is non-abelian, for otherwise, $G=C$ by $[4$, Theorem $4.2 \mathrm{~A}]$. Let $H$ be a core-free subgroup of $G$ that affords the minimal faithful representation. By Theorem 2.3, $C \cong N_{G}(H) / H$ and since $|G: H|=9, \mid N_{G}(H)$ : $H \mid=3$ and so $C \cong C_{3}$. By Proposition $2.5, \operatorname{Fix}(H)$ is a block on which the induced permutation group acts regularly, so by Proposition 2.6, $G$ embeds inside the wreath product $C_{3} 2 \operatorname{Sym}(3)$. Let $\pi$ be the projection of $G$ onto the top group $\operatorname{Sym}(3)$. Now ker $\pi$ is contained in the base group and so must be a 3 -group. Since $G$ is transitive on blocks, $G \pi$ has order 3 or 6 . If $|G \pi|=3$, then $G$ is a 3 -group and so, by Corollary $2.12, C \leq G$. If $|G \pi|=6$ then $\pi$ is surjective and since ker $\pi$ is a normal subgroup of $G$ contained in the base group $B$ it is normalised by $\operatorname{Sym}(3)$. By Lemma 3.10, this kernel must contain $V$, which is cyclic of order 3 and central in $G$. Therefore $V=C$, and once again $C \leq G$.

Remark 3.12. It can be verified that there are, up to isomorphism, 3 transitive groups minimally embedded in $\operatorname{Sym}(9)$ not contained in $\mathscr{C}$. Again, we do not prove this here as we do not need it for our main theorem, and for more details the reader is referred to [8].

Combining the results above we can now prove our main theorem which we restate here for convenience: 
Theorem 3.13. If $G$ and $H$ are groups such that $\mu(G \times H) \leq 9$ then $\mu(G \times H)=\mu(G)+$ $\mu(H)$.

Proof. Suppose by way of contradiction that there exist nontrivial subgroups $G, H$ of $\operatorname{Sym}(9)$ such that $\langle G, H\rangle=G H$ is an internal direct product and $\mu(G \times H)<\mu(G)+\mu(H)$. Without loss of generality, we may suppose $G \notin \mathscr{C}$. By Proposition $3.1, \mu(G) \geq 7$. If $\mu(G)=9$, then, by Proposition 3.8 and Proposition 3.11, $H$ intersects $G$ nontrivially, contradicting that $G H$ is an internal direct product. Hence $\mu(G)=7$ or $\mu(G)=8$.

Suppose that $\mu(G)=7$. By Theorem 3.4 and $(2), G \cong\left\langle a, b \mid a^{3}=b^{4}=1, a^{b}=a^{-1}\right\rangle$. Without loss of generality, we may take $a=(123),(123)(456)$ or (123)(456)(789), and $b$ to be a permutation of order 4 that inverts $a$ by conjugation. By straightforward calculations, the only permutations in $\operatorname{Sym}(9)$ that invert (123)(456) or (1 23)(456)(789) have order 2 or 6 , contradicting that $b$ has order 4 . Hence $a=(123)$. Without loss of generality, $b=(13)(4567)$ or $(13)(4567)(89)$ and $G=\langle a, b\rangle$. Clearly, in either case,

$$
C_{\text {Sym }(9)}(G)=\langle(4567),(89)\rangle .
$$

But $G H$ is an internal direct product, so $H \cap G=\{1\}$ and $H \leq C_{\operatorname{Sym}(9)}(G)$. Since $H$ is non-trivial, it follows that $H=\langle(89)\rangle$ or $H=\langle(46)(57)(89)\rangle$. In both cases, $H \cong C_{2}$ so $\mu(H)=2$, giving

$$
\mu(G \times H)<\mu(G)+\mu(H)=7+2=9 .
$$

Hence $\mu(G \times H) \leq 8$, contradicting Theorem 3.7.

Thus $\mu(G)=8$. By Theorem 3.6, $G$ contains a copy of the group $K \cong\left(C_{2} \times C_{2} \times C_{2}\right) \rtimes C_{7}$ described explicitly in the preamble preceding Proposition 3.5. All elements of the base group different from 1 are conjugate in $K$. We may choose generators of the base group to be $x, y$ and $z$ and the generator corresponding to the copy of $C_{7}$ to be $t$, such that conjugation by $t$ yields the following mapping:

$$
x \mapsto y \mapsto z \mapsto x y \mapsto y z \mapsto x y z \mapsto x z \mapsto x .
$$

Suppose first that $x$ is not a product of 4 disjoint 2-cycles. Without loss of generality we have the following three cases.

Case (i): $x=(12)$.

Then $y=(a b)$ commutes with $x$ and so is disjoint from $x$, and so $x y=(12)(a b)$ is not conjugate to $x$.

Case (ii): $x=(12)(34)$.

Without loss of generality, $y=(13)(24),(12)(56)$ or $(56)(78)$. If $y=(56)(78)$ then $x y=(12)(34)(56)(78)$ is not conjugate to $x$. If $y=(13)(24)$ then, without loss of generality, $z=(56)(78)$, so $x z$ is not conjugate to $x$. If $y=(12)(56)$ then $z=(12)(78),(34)(78)$ or $(56)(78)$ so that $(12)(34)(56)(78)=x y z, y z$ or $x z$ respectively is not conjugate to $x$.

Case (iii): $x=(12)(34)(56)$.

Without loss of generality, $y=(12)(34)(78),(13)(24)(56)$ or $(13)(24)(78)$. Then $x y=$ $(56)(78),(14)(23)$ or $(14)(23)(56)(78)$ respectively is not conjugate to $x$. 
All of these cases lead to a contradiction, so, without loss of generality, $x=(12)(34)(56)(78)$. Now both $y$ and $z$ fix 9 since $x=x^{y}=x^{z}$. If $t$ moves 9 , then $x^{t}$ moves 9 , contradicting that $x^{t}=y$ fixes 9 . Hence $t$ also fixes 9 and so $t$ is a 7 -cycle permuting letters amongst $\{1, \ldots, 8\}$. Therefore, $t$ fixes another letter and so, without loss of generality, $t$ is a 7-cycle permuting $1, \ldots, 7$ in some order. Let $w \in C:=C_{\operatorname{Sym}(9)}(G)$. Then $w$ commutes with $t$, so $w=t^{r}$, or $w=t^{r}(89)$ for some $r$. But if $w=t^{r}(89)$, then $x^{w}$ moves 9 , so $x^{w} \neq x$, contradicting that $w$ commutes with $x$. Hence $w=t^{r}$, which implies $w=1$, since non-trivial powers of $t$ do not commute with $x$. Thus $H \leq C=\{1\}$, so $H$ is trivial, a contradiction. This completes the proof of the theorem.

The string of results above show that there are no examples of groups $G$ and $H$ such that $\mu(G \times H) \leq 9$ and

$$
\mu(G \times H)<\mu(G)+\mu(H) .
$$

In $\operatorname{Sym}(10)$, however, $G$ can be taken to be any split extension of the deleted permutation module for $\operatorname{Sym}(5)$ over $\mathbb{F}_{2}$ by a subgroup that contains an element of order 5 . It is well-known that there are 5 such choices for the top group of the split extension, namely $C_{5}, D_{10}, C_{5} \rtimes C_{4}, \operatorname{Alt}(5)$ or $\operatorname{Sym}(5)$. So, for the example of smallest order, one takes $G$ to be $\left(C_{2} \times C_{2} \times C_{2} \times C_{2}\right) \rtimes C_{5}$ and $H$ to be $C_{2}$ (its centraliser in Sym(10)), and all examples have the property that $\mu(G)=\mu(G \times H)<\mu(G)+\mu(H)$.

The authors are not aware of any examples of groups $G$ and $H$ that do not decompose as nontrivial direct products for which

$$
\max \{\mu(G), \mu(H)\}<\mu(G \times H)<\mu(G)+\mu(H) .
$$

One can easily transform (4) into an infinite class of examples of (5) by taking direct products with a new group of order coprime to both $G$ and $H$ (see $[9$, Section 7$]$ ).

\section{ACKNOWLEDGEMENTS}

The authors thank Cheryl Praeger for helpful discussions in catalysing the proofs in some of the results above, particularly in the transitive cases. The authors thank the referee for his or her careful reading of the manuscript and suggestions leading to significant corrections and improvements, especially for suggesting a shortened proof of Theorem 3.6.

Part of this work was completed while the second author was on a research visit to the University of Sydney. He sincerely thanks Anthony Henderson for supporting this research visit. 
Appendix A.

Minimal Degrees of Groups of Small Order

Below is a table of minimal permutation degrees of groups of small order which we rely on in the article. Many of these calculations can be done by hand, or can easily be verified using Magma [1]. The group identification is done using the SmallGroupsLibrary in Magma.

Table 1: Minimal Degrees of Groups of Small Order

\begin{tabular}{|c|c|c|c|}
\hline Group ID & Stucture & Minimal Degree & Contained in $\mathscr{C} ?$ \\
\hline$(56,1)$ & {$\left[C_{7}: C_{8}\right]$} & 15 & $\mathrm{No}$ \\
\hline$(56,2)$ & {$\left[C_{56}\right]$} & 15 & Yes Abelian \\
\hline$(56,3)$ & {$\left[C_{7}: Q_{8}\right]$} & 15 & No \\
\hline$(56,4)$ & {$\left[C_{4} \times D_{14}\right]$} & 11 & Yes \\
\hline$(56,5)$ & {$\left[D_{56}\right]$} & 11 & Yes \\
\hline$(56,6)$ & {$\left[C_{2} \times\left(C_{7}: C_{4}\right)\right]$} & 13 & No \\
\hline$(56,7)$ & {$\left[\left(C_{14} \times C_{2}\right): C_{2}\right]$} & 11 & Yes \\
\hline$(56,8)$ & {$\left[C_{28} \times C_{2}\right]$} & 13 & Yes Abelian \\
\hline$(56,9)$ & {$\left[C_{7} \times D_{8}\right]$} & 11 & Yes Nilpotent \\
\hline$(56,10)$ & {$\left[C_{7} \times Q_{8}\right]$} & 15 & Yes Nilpotent \\
\hline$(56,11)$ & {$\left[\left(C_{2} \times C_{2} \times C_{2}\right): C_{7}\right]$} & 8 & No \\
\hline$(56,12)$ & {$\left[C_{2} \times C_{2} \times D_{14}\right]$} & 11 & Yes \\
\hline$(56,13)$ & {$\left[C_{14} \times C_{2} \times C_{2}\right]$} & 13 & Yes Abelian \\
\hline$(54,1)$ & {$\left[D_{54}\right]$} & 27 & Yes \\
\hline$(54,2)$ & {$\left[C_{54}\right]$} & 29 & Yes Abelian \\
\hline$(54,3)$ & {$\left[C_{3} \times D_{18}\right]$} & 12 & Yes \\
\hline$(54,4)$ & {$\left[C_{9} \times \operatorname{Sym}(3)\right]$} & 12 & Yes \\
\hline$(54,5)$ & {$\left[\left(\left(C_{3} \times C_{3}\right): C_{3}\right): C_{2}\right]$} & 9 & Yes \\
\hline$(54,6)$ & {$\left[\left(C_{9}: C_{3}\right): C_{2}\right]$} & 9 & Yes \\
\hline$(54,7)$ & {$\left[\left(C_{9} \times C_{3}\right): C_{2}\right]$} & 12 & Yes \\
\hline$(54,8)$ & {$\left[\left(\left(C_{3} \times C_{3}\right): C_{3}\right): C_{2}\right]$} & 9 & Yes \\
\hline$(54,9)$ & {$\left[C_{18} \times C_{3}\right]$} & 14 & Yes Abelian \\
\hline$(54,10)$ & {$\left[C_{2} \times\left(\left(C_{3} \times C_{3}\right): C_{3}\right)\right]$} & 11 & Yes Nilpotent \\
\hline$(54,11)$ & {$\left[C_{2} \times\left(C_{9}: C_{3}\right)\right]$} & 11 & Yes Nilpotent \\
\hline$(54,12)$ & {$\left[C_{3} \times C_{3} \times \operatorname{Sym}(3)\right]$} & 9 & Yes \\
\hline$(54,13)$ & {$\left[C_{3} \times\left(\left(C_{3} \times C_{3}\right): C_{2}\right)\right]$} & 9 & Yes \\
\hline$(54,14)$ & {$\left[\left(C_{3} \times C_{3} \times C_{3}\right): C_{2}\right]$} & 9 & Yes \\
\hline$(54,15)$ & {$\left[C_{6} \times C_{3} \times C_{3}\right]$} & 11 & Yes Abelian \\
\hline$(48,1)$ & {$\left[C_{3}: C_{16}\right]$} & 19 & No \\
\hline$(48,2)$ & {$\left[C_{48}\right]$} & 19 & Yes Abelian \\
\hline$(48,3)$ & {$\left[\left(C_{4} \times C_{4}\right): C_{3}\right]$} & 12 & No \\
\hline$(48,4)$ & {$\left[C_{8} \times \operatorname{Sym}(3)\right]$} & 11 & Yes \\
\hline$(48,5)$ & {$\left[C_{24}: C_{2}\right]$} & 11 & Yes \\
\hline$(48,6)$ & {$\left[C_{24}: C_{2}\right]$} & 11 & Yes \\
\hline
\end{tabular}


Table 1 - Continued from previous page

\begin{tabular}{|c|c|c|c|}
\hline Group ID & Structure & Minimal Degree & Contained in $\mathscr{C} ?$ \\
\hline$(48,7)$ & {$\left[D_{48}\right]$} & 11 & Yes \\
\hline$(48,8)$ & {$\left[C_{3}: Q_{16}\right]$} & 19 & No \\
\hline$(48,9)$ & {$\left[C_{2} \times\left(C_{3}: C_{8}\right)\right]$} & 13 & No \\
\hline$(48,10)$ & {$\left[\left(C_{3}: C_{8}\right): C_{2}\right]$} & 11 & No \\
\hline$(48,11)$ & {$\left[C_{4} \times\left(C_{3}: C_{4}\right)\right]$} & 11 & No \\
\hline$(48,12)$ & {$\left[\left(C_{3}: C_{4}\right): C_{4}\right]$} & 11 & No \\
\hline$(48,13)$ & {$\left[C_{12}: C_{4}\right]$} & 11 & No \\
\hline$(48,14)$ & {$\left[\left(C_{12} \times C_{2}\right): C_{2}\right]$} & 11 & No \\
\hline$(48,15)$ & {$\left[\left(C_{3} \times D_{8}\right): C_{2}\right]$} & 11 & No \\
\hline$(48,16)$ & {$\left[\left(C_{3}: C_{8}\right): C_{2}\right]$} & 11 & No \\
\hline$(48,17)$ & {$\left[\left(C_{3} \times Q_{8}\right): C_{2}\right]$} & 11 & No \\
\hline$(48,18)$ & {$\left[C_{3}: Q_{16}\right]$} & 19 & No \\
\hline$(48,19)$ & {$\left[\left(C_{2} \times\left(C_{3}: C_{4}\right)\right): C_{2}\right]$} & 11 & No \\
\hline$(48,20)$ & {$\left[C_{12} \times C_{4}\right]$} & 11 & Yes Abelian \\
\hline$(48,21)$ & {$\left[C_{3} \times\left(\left(C_{4} \times C_{2}\right): C_{2}\right)\right]$} & 11 & Yes Nilpotent \\
\hline$(48,22)$ & {$\left[C_{3} \times\left(C_{4}: C_{4}\right)\right]$} & 11 & Yes Nilpotent \\
\hline$(48,23)$ & {$\left[C_{24} \times C_{2}\right]$} & 13 & Yes Abelian \\
\hline$(48,24)$ & {$\left[C_{3} \times\left(C_{8}: C_{2}\right)\right]$} & 11 & Yes Nilpotent \\
\hline$(48,25)$ & {$\left[C_{3} \times D_{16}\right]$} & 11 & Yes \\
\hline$(48,26)$ & {$\left[C_{3} \times Q D_{16}\right]$} & 11 & Yes \\
\hline$(48,27)$ & {$\left[C_{3} \times Q_{16}\right]$} & 19 & Yes Nilpotent \\
\hline$(48,28)$ & {$\left[S L(2,3) \rightarrow G \rightarrow C_{2}\right]$} & 16 & Yes \\
\hline$(48,29)$ & {$[G L(2,3)]$} & 8 & Yes \\
\hline$(48,30)$ & {$\left[\operatorname{Alt}(4): C_{4}\right]$} & 8 & Yes \\
\hline$(48,31)$ & {$\left[C_{4} \times \operatorname{Alt}(4)\right]$} & 8 & Yes \\
\hline$(48,32)$ & {$\left[C_{2} \times S L(2,3)\right]$} & 10 & Yes \\
\hline$(48,33)$ & {$\left[S L(2,3): C_{2}\right]$} & 16 & No \\
\hline$(48,34)$ & {$\left[C_{2} \times\left(C_{3}: Q_{8}\right)\right]$} & 13 & No \\
\hline$(48,35)$ & {$\left[C_{2} \times C_{4} \times \operatorname{Sym}(3)\right]$} & 9 & Yes \\
\hline$(48,36)$ & {$\left[C_{2} \times D_{24}\right]$} & 9 & Yes \\
\hline$(48,37)$ & {$\left[\left(C_{12} \times C_{2}\right): C_{2}\right]$} & 11 & No \\
\hline$(48,38)$ & {$\left[D_{8} \times \operatorname{Sym}(3)\right]$} & 7 & Yes \\
\hline$(48,39)$ & {$\left[\left(C_{2} \times\left(C_{3}: C_{4}\right)\right): C_{2}\right]$} & 11 & No \\
\hline$(48,40)$ & {$\left[Q_{8} \times \operatorname{Sym}(3)\right]$} & 11 & Yes \\
\hline$(48,41)$ & {$\left[\left(C_{4} \times \operatorname{Sym}(3)\right): C_{2}\right]$} & 11 & Yes \\
\hline$(48,42)$ & {$\left[C_{2} \times C_{2} \times\left(C_{3}: C_{4}\right)\right]$} & 11 & No \\
\hline$(48,43)$ & {$\left[C_{2} \times\left(\left(C_{6} \times C_{2}\right): C_{2}\right)\right]$} & 9 & Yes \\
\hline$(48,44)$ & {$\left[C_{12} \times C_{2} \times C_{2}\right]$} & 11 & Yes Abelian \\
\hline$(48,45)$ & {$\left[C_{6} \times D_{8}\right]$} & 9 & Yes Nilpotent \\
\hline$(48,46)$ & {$\left[C_{6} \times Q_{8}\right]$} & 13 & Yes Nilpotent \\
\hline
\end{tabular}


Table 1 - Continued from previous page

\begin{tabular}{|c|c|c|c|}
\hline Group ID & Structure & Minimal Degree & Contained in $\mathscr{C} ?$ \\
\hline$(48,47)$ & {$\left[C_{3} \times\left(\left(C_{4} \times C_{2}\right): C_{2}\right)\right]$} & 11 & Yes Nilpotent \\
\hline$(48,48)$ & {$\left[C_{2} \times \operatorname{Sym}(4)\right]$} & 6 & Yes \\
\hline$(48,49)$ & {$\left[C_{2} \times C_{2} \times \operatorname{Alt}(4)\right]$} & 8 & Yes \\
\hline$(48,50)$ & {$\left[\left(C_{2} \times C_{2} \times C_{2} \times C_{2}\right): C_{3}\right]$} & 8 & Yes \\
\hline$(48,51)$ & {$\left[C_{2} \times C_{2} \times C_{2} \times \operatorname{Sym}(3)\right]$} & 9 & Yes \\
\hline$(48,52)$ & {$\left[C_{6} \times C_{2} \times C_{2} \times C_{2}\right]$} & 11 & Yes Abelian \\
\hline$(40,1)$ & {$\left[C_{5}: C_{8}\right]$} & 13 & No \\
\hline$(40,2)$ & {$\left[C_{40}\right]$} & 13 & Yes Abelian \\
\hline$(40,3)$ & {$\left[C_{5}: C_{8}\right]$} & 13 & No \\
\hline$(40,4)$ & {$\left[C_{5}: Q_{8}\right]$} & 13 & No \\
\hline$(40,5)$ & {$\left[C_{4} \times D_{10}\right]$} & 9 & Yes \\
\hline$(40,6)$ & {$\left[D_{40}\right]$} & 9 & Yes \\
\hline$(40,7)$ & {$\left[C_{2} \times\left(C_{5}: C_{4}\right)\right]$} & 11 & No \\
\hline$(40,8)$ & {$\left[\left(C_{10} \times C_{2}\right): C_{2}\right]$} & 9 & Yes \\
\hline$(40,9)$ & {$\left[C_{20} \times C_{2}\right]$} & 11 & Yes Abelian \\
\hline$(40,10)$ & {$\left[C_{5} \times D_{8}\right]$} & 9 & Yes Nilpotent \\
\hline$(40,11)$ & {$\left[C_{5} \times Q_{8}\right]$} & 13 & Yes Nilpotent \\
\hline$(40,12)$ & {$\left[C_{2} \times\left(C_{5}: C_{4}\right)\right]$} & 7 & Yes \\
\hline$(40,13)$ & {$\left[C_{2} \times C_{2} \times D_{10}\right]$} & 9 & Yes \\
\hline$(40,14)$ & {$\left[C_{10} \times C_{2} \times C_{2}\right]$} & 11 & Yes Abelian \\
\hline$(36,1)$ & {$\left[C_{9}: C_{4}\right]$} & 13 & No \\
\hline$(36,2)$ & {$\left[C_{36}\right]$} & 13 & Yes Abelian \\
\hline$(36,3)$ & {$\left[\left(C_{2} \times C_{2}\right): C_{9}\right]$} & 13 & No \\
\hline$(36,4)$ & {$\left[D_{36}\right]$} & 11 & Yes \\
\hline$(36,5)$ & {$\left[C_{18} \times C_{2}\right]$} & 13 & Yes Abelian \\
\hline$(36,6)$ & {$\left[C_{3} \times\left(C_{3}: C_{4}\right)\right]$} & 10 & No \\
\hline$(36,7)$ & {$\left[\left(C_{3} \times C_{3}\right): C_{4}\right]$} & 10 & No \\
\hline$(36,8)$ & {$\left[C_{12} \times C_{3}\right]$} & 10 & Yes Abelian \\
\hline$(36,9)$ & {$\left[\left(C_{3} \times C_{3}\right): C_{4}\right]$} & 6 & Yes \\
\hline$(36,10)$ & {$[\operatorname{Sym}(3) \times \operatorname{Sym}(3)]$} & 6 & Yes \\
\hline$(36,11)$ & {$\left[C_{3} \times \operatorname{Alt}(4)\right]$} & 7 & Yes \\
\hline$(36,12)$ & {$\left[C_{6} \times \operatorname{Sym}(3)\right]$} & 8 & Yes \\
\hline$(36,13)$ & {$\left[C_{2} \times\left(\left(C_{3} \times C_{3}\right): C_{2}\right)\right]$} & 8 & Yes \\
\hline$(36,14)$ & {$\left[C_{6} \times C_{6}\right]$} & 10 & Yes Abelian \\
\hline$(32,1)$ & {$\left[C_{32}\right]$} & 32 & Yes Abelian \\
\hline$(32,2)$ & {$\left[\left(C_{4} \times C_{2}\right): C_{4}\right]$} & 12 & Yes Nilpotent \\
\hline$(32,3)$ & {$\left[C_{8} \times C_{4}\right]$} & 12 & Yes Abelian \\
\hline$(32,4)$ & {$\left[C_{8}: C_{4}\right]$} & 12 & Yes Nilpotent \\
\hline$(32,5)$ & {$\left[\left(C_{8} \times C_{2}\right): C_{2}\right]$} & 12 & Yes Nilpotent \\
\hline$(32,6)$ & {$\left[\left(\left(C_{4} \times C_{2}\right): C_{2}\right): C_{2}\right]$} & 8 & Yes Nilpotent \\
\hline
\end{tabular}


Table 1 - Continued from previous page

\begin{tabular}{|c|c|c|c|}
\hline Group ID & Structure & Minimal Degree & Contained in $\mathscr{C} ?$ \\
\hline$(32,7)$ & {$\left[\left(C_{8}: C_{2}\right): C_{2}\right]$} & 8 & Yes Nilpotent \\
\hline$(32,8)$ & {$\left[C_{2} \cdot\left(\left(C_{4} \times C_{2}\right): C_{2}\right)=\left(C_{2} \times C_{2}\right) \cdot\left(C_{4} \times C_{2}\right)\right]$} & 16 & Yes Nilpotent \\
\hline$(32,9)$ & {$\left[\left(C_{8} \times C_{2}\right): C_{2}\right]$} & 12 & Yes Nilpotent \\
\hline$(32,10)$ & {$\left[Q_{8}: C_{4}\right]$} & 12 & Yes Nilpotent \\
\hline$(32,11)$ & {$\left[\left(C_{4} \times C_{4}\right): C_{2}\right]$} & 8 & Yes Nilpotent \\
\hline$(32,12)$ & {$\left[C_{4}: C_{8}\right]$} & 12 & Yes Nilpotent \\
\hline$(32,13)$ & {$\left[C_{8}: C_{4}\right]$} & 12 & Yes Nilpotent \\
\hline$(32,14)$ & {$\left[C_{8}: C_{4}\right]$} & 12 & Yes Nilpotent \\
\hline$(32,15)$ & {$\left[C_{4} \cdot D_{8}=C_{4} \cdot\left(C_{4} \times C_{2}\right)\right]$} & 16 & Yes Nilpotent \\
\hline$(32,16)$ & {$\left[C_{16} \times C_{2}\right]$} & 18 & Yes Abelian \\
\hline$(32,17)$ & {$\left[C_{16}: C_{2}\right]$} & 16 & Yes Nilpotent \\
\hline$(32,18)$ & {$\left[D_{32}\right]$} & 16 & Yes Nilpotent \\
\hline$(32,19)$ & {$\left[Q D_{32}\right]$} & 16 & Yes Nilpotent \\
\hline$(32,20)$ & {$\left[Q_{32}\right]$} & 32 & Yes Nilpotent \\
\hline$(32,21)$ & {$\left[C_{4} \times C_{4} \times C_{2}\right]$} & 10 & Yes Abelian \\
\hline$(32,22)$ & {$\left[C_{2} \times\left(\left(C_{4} \times C_{2}\right): C_{2}\right)\right]$} & 10 & Yes Nilpotent \\
\hline$(32,23)$ & {$\left[C_{2} \times\left(C_{4}: C_{4}\right)\right]$} & 10 & Yes Nilpotent \\
\hline$(32,24)$ & {$\left[\left(C_{4} \times C_{4}\right): C_{2}\right]$} & 12 & Yes Nilpotent \\
\hline$(32,25)$ & {$\left[C_{4} \times D_{8}\right]$} & 8 & Yes Nilpotent \\
\hline$(32,26)$ & {$\left[C_{4} \times Q_{8}\right]$} & 12 & Yes Nilpotent \\
\hline$(32,27)$ & {$\left[\left(C_{2} \times C_{2} \times C_{2} \times C_{2}\right): C_{2}\right]$} & 8 & Yes Nilpotent \\
\hline$(32,28)$ & {$\left[\left(C_{4} \times C_{2} \times C_{2}\right): C_{2}\right]$} & 8 & Yes Nilpotent \\
\hline$(32,29)$ & {$\left[\left(C_{2} \times Q_{8}\right): C_{2}\right]$} & 12 & Yes Nilpotent \\
\hline$(32,30)$ & {$\left[\left(C_{4} \times C_{2} \times C_{2}\right): C_{2}\right]$} & 12 & Yes Nilpotent \\
\hline$(32,31)$ & {$\left[\left(C_{4} \times C_{4}\right): C_{2}\right]$} & 12 & Yes Nilpotent \\
\hline$(32,32)$ & {$\left[\left(C_{2} \times C_{2}\right) \cdot\left(C_{2} \times C_{2} \times C_{2}\right)\right]$} & 16 & Yes Nilpotent \\
\hline$(32,33)$ & {$\left[\left(C_{4} \times C_{4}\right): C_{2}\right]$} & 16 & Yes Nilpotent \\
\hline$(32,34)$ & {$\left[\left(C_{4} \times C_{4}\right): C_{2}\right]$} & 8 & Yes Nilpotent \\
\hline$(32,35)$ & {$\left[C_{4}: Q_{8}\right]$} & 12 & Yes Nilpotent \\
\hline$(32,36)$ & {$\left[C_{8} \times C_{2} \times C_{2}\right]$} & 12 & Yes Abelian \\
\hline$(32,37)$ & {$\left[C_{2} \times\left(C_{8}: C_{2}\right)\right]$} & 10 & Yes Nilpotent \\
\hline$(32,38)$ & {$\left[\left(C_{8} \times C_{2}\right): C_{2}\right]$} & 16 & Yes Nilpotent \\
\hline$(32,39)$ & {$\left[C_{2} \times D_{16}\right]$} & 10 & Yes Nilpotent \\
\hline$(32,40)$ & {$\left[C_{2} \times Q D_{16}\right]$} & 10 & Yes Nilpotent \\
\hline$(32,41)$ & {$\left[C_{2} \times Q_{16}\right]$} & 18 & Yes Nilpotent \\
\hline$(32,42)$ & {$\left[\left(C_{8} \times C_{2}\right): C_{2}\right]$} & 16 & Yes Nilpotent \\
\hline$(32,43)$ & {$\left[\left(C_{2} \times D_{8}\right): C_{2}\right]$} & 8 & Yes Nilpotent \\
\hline$(32,44)$ & {$\left[\left(C_{2} \times Q_{8}\right): C_{2}\right]$} & 16 & Yes Nilpotent \\
\hline$(32,45)$ & {$\left[C_{4} \times C_{2} \times C_{2} \times C_{2}\right]$} & 10 & Yes Abelian \\
\hline$(32,46)$ & {$\left[C_{2} \times C_{2} \times D_{8}\right]$} & 8 & Yes Nilpotent \\
\hline
\end{tabular}


Table 1 - Continued from previous page

\begin{tabular}{|c|c|c|c|}
\hline Group ID & Structure & Minimal Degree & Contained in $\mathscr{C} ?$ \\
\hline$(32,47)$ & {$\left[C_{2} \times C_{2} \times Q_{8}\right]$} & 12 & Yes Nilpotent \\
\hline$(32,48)$ & {$\left[C_{2} \times\left(\left(C_{4} \times C_{2}\right): C_{2}\right)\right]$} & 10 & Yes Nilpotent \\
\hline$(32,49)$ & {$\left[\left(C_{2} \times D_{8}\right): C_{2}\right]$} & 8 & Yes Nilpotent \\
\hline$(32,50)$ & {$\left[\left(C_{2} \times Q_{8}\right): C_{2}\right]$} & 16 & Yes Nilpotent \\
\hline$(32,51)$ & {$\left[C_{2} \times C_{2} \times C_{2} \times C_{2} \times C_{2}\right]$} & 10 & Yes Abelian \\
\hline$(28,1)$ & {$\left[C_{7}: C_{4}\right]$} & 11 & No \\
\hline$(28,2)$ & {$\left[C_{28}\right]$} & 11 & Yes Abelian \\
\hline$(28,3)$ & {$\left[D_{28}\right]$} & 9 & Yes \\
\hline$(28,4)$ & {$\left[C_{14} \times C_{2}\right]$} & 11 & Yes Abelian \\
\hline$(27,1)$ & {$\left[C_{27}\right]$} & 27 & Yes Abelian \\
\hline$(27,2)$ & {$\left[C_{9} \times C_{3}\right]$} & 12 & Yes Abelian \\
\hline$(27,3)$ & {$\left[\left(C_{3} \times C_{3}\right): C_{3}\right]$} & 9 & Yes Nilpotent \\
\hline$(27,4)$ & {$\left[C_{9}: C_{3}\right]$} & 9 & Yes Nilpotent \\
\hline$(27,5)$ & {$\left[C_{3} \times C_{3} \times C_{3}\right]$} & 9 & Yes Abelian \\
\hline$(24,1)$ & {$\left[C_{3}: C_{8}\right]$} & 11 & No \\
\hline$(24,2)$ & {$\left[C_{24}\right]$} & 11 & Yes Abelian \\
\hline$(24,3)$ & {$[S L(2,3)]$} & 8 & Yes \\
\hline$(24,4)$ & {$\left[C_{3}: Q_{8}\right]$} & 11 & No \\
\hline$(24,5)$ & {$\left[C_{4} \times \operatorname{Sym}(3)\right]$} & 7 & Yes \\
\hline$(24,6)$ & {$\left[D_{24}\right]$} & 7 & Yes \\
\hline$(24,7)$ & {$\left[C_{2} \times\left(C_{3}: C_{4}\right)\right]$} & 9 & No \\
\hline$(24,8)$ & {$\left[\left(C_{6} \times C_{2}\right): C_{2}\right]$} & 7 & Yes \\
\hline$(24,9)$ & {$\left[C_{12} \times C_{2}\right]$} & 9 & Yes Abelian \\
\hline$(24,10)$ & {$\left[C_{3} \times D_{8}\right]$} & 7 & Yes Nilpotent \\
\hline$(24,11)$ & {$\left[C_{3} \times Q_{8}\right]$} & 11 & Yes Nilpotent \\
\hline$(24,12)$ & {$[\operatorname{Sym}(4)]$} & 4 & Yes \\
\hline$(24,13)$ & {$\left[C_{2} \times \operatorname{Alt}(4)\right]$} & 6 & Yes \\
\hline$(24,14)$ & {$\left[C_{2} \times C_{2} \times \operatorname{Sym}(3)\right]$} & 7 & Yes \\
\hline$(24,15)$ & {$\left[C_{6} \times C_{2} \times C_{2}\right]$} & 9 & Yes Abelian \\
\hline$(20,1)$ & {$\left[C_{5}: C_{4}\right]$} & 9 & No \\
\hline$(20,2)$ & {$\left[C_{20}\right]$} & 9 & Yes Abelian \\
\hline$(20,3)$ & {$\left[C_{5}: C_{4}\right]$} & 5 & Yes \\
\hline$(20,4)$ & {$\left[D_{20}\right]$} & 7 & Yes \\
\hline$(20,5)$ & {$\left[C_{10} \times C_{2}\right]$} & 9 & Yes Abelian \\
\hline$(18,1)$ & {$\left[D_{18}\right]$} & 9 & Yes \\
\hline$(18,2)$ & {$\left[C_{18}\right]$} & 11 & Yes Abelian \\
\hline$(18,3)$ & {$\left[C_{3} \times \operatorname{Sym}(3)\right]$} & 6 & Yes \\
\hline$(18,4)$ & $\left.\left(C_{3} \times C_{3}\right): C_{2}\right]$ & 6 & Yes \\
\hline$(18,5)$ & {$\left[C_{6} \times C_{3}\right]$} & 8 & Yes Abelian \\
\hline$(16,1)$ & {$\left[C_{16}\right]$} & 16 & Yes Abelian \\
\hline
\end{tabular}


Table 1 - Continued from previous page

\begin{tabular}{|l|l|c|l|}
\hline Group ID & Structure & Minimal Degree & Contained in $\mathscr{C} ?$ \\
\hline$(16,2)$ & {$\left[C_{4} \times C_{4}\right]$} & 8 & Yes Abelian \\
$(16,3)$ & {$\left[\left(C_{4} \times C_{2}\right): C_{2}\right]$} & 8 & Yes Nilpotent \\
$(16,4)$ & {$\left[C_{4}: C_{4}\right]$} & 8 & Yes Nilpotent \\
$(16,5)$ & {$\left[C_{8} \times C_{2}\right]$} & 10 & Yes Abelian \\
$(16,6)$ & {$\left[C_{8}: C_{2}\right]$} & 8 & Yes Nilpotent \\
$(16,7)$ & {$\left[D_{16}\right]$} & 8 & Yes Nilpotent \\
$(16,8)$ & {$\left[Q D_{16}\right]$} & 8 & Yes Nilpotent \\
$(16,9)$ & {$\left[Q_{16}\right]$} & 16 & Yes Nilpotent \\
$(16,10)$ & {$\left[C_{4} \times C_{2} \times C_{2}\right]$} & 8 & Yes Abelian \\
$(16,11)$ & {$\left[C_{2} \times D_{8}\right]$} & 6 & Yes Nilpotent \\
$(16,12)$ & {$\left[C_{2} \times Q_{8}\right]$} & 10 & Yes Nilpotent \\
$(16,13)$ & {$\left[\left(C_{4} \times C_{2}\right): C_{2}\right]$} & 8 & Yes Nilpotent \\
$(16,14)$ & {$\left[C_{2} \times C_{2} \times C_{2} \times C_{2}\right]$} & 8 & Yes Abelian \\
$(12,1)$ & {$\left[C_{3}: C_{4}\right]$} & 7 & No \\
$(12,2)$ & {$\left[C_{12}\right]$} & 7 & Yes Abelian \\
$(12,3)$ & {$[$ Alt $(4)]$} & 4 & Yes \\
$(12,4)$ & {$\left[D_{12}\right]$} & 5 & Yes \\
$(12,5)$ & {$\left[C_{6} \times C_{2}\right]$} & 7 & Yes Abelian \\
\hline
\end{tabular}




\section{REFERENCES}

[1] W. Bosma, J.J. Cannon, C. Fieker, and A. Steel. (Eds.) Handbook of Magma Functions,. Edition 2.16, 5017 pages, 2010.

[2] G. Butler and J. McKay. The transitive groups of degree up to eleven. Comm. Algebra 11:863-911, 1983.

[3] P.J. Cameron. Permutation Groups. Student Texts 45, London Math. Soc., Cambridge University Press, 1999.

[4] J.D. Dixon and B. Mortimer. Permutation Groups. Springer-Verlag New York, 1996.

[5] D. Easdown and C.E. Praeger. On minimal faithful permutation representations of finite groups. Bull. Austral. Math. Soc., 38:207-220, 1988.

[6] D.L. Johnson. Minimal permutation representations of finite groups. Amer. J. Math., 93(4):857-866, 1971.

[7] N. Saunders. The minimal degree for a class of finite complex reflection groups. J. Algebra, 323:561-573, 2010.

[8] N. Saunders. Minimal Faithful Permutation Representations of Finite Groups. Ph.D. Thesis, University of Sydney, 2011.

[9] N. Saunders. Minimal faithful permutation degrees for irreducible coxeter groups and binary polyhedral groups. J. Group Theory, 17(5):805-832, 2014.

[10] D. Wright. Degrees of minimal embeddings of some direct products. Amer. J. Math., 97:897-903, 1975.

School of Mathematics and Statistics, University of Sydney, NSW 2006, Australia

E-mail address: david.easdown@sydney.edu.au

Heilbronn Institute for Mathematical Research, University of Bristol, School of Mathematics, University Walk, Bristol BS8 1TW, United Kingdom

E-mail address: neil.saunders@bristol.ac.uk 